\title{
Percutaneous cementoplasty for painful osteolytic distal femur metastases: a case report
}

\author{
Mingxing Lei' \\ Yaosheng Liu' \\ Shaoxing Yang ${ }^{2}$ \\ Weigang Jiang' \\ Yuncen Cao' \\ Shubin Liu' \\ 'Department of Orthopedic \\ Surgery, ${ }^{2}$ Department of Pulmonary \\ Neoplasms Internal Medicine, The \\ Affiliated Hospital of Academy of \\ Military Medical Sciences, Beijing, \\ People's Republic of China
}

This article was published in the following Dove Press journal: Journal of Pain Research

19 October 2016

Number of times this article has been viewed
Correspondence: Yaosheng Liu; Shubin Liu Department of Orthopedic Surgery, The Affiliated Hospital of Academy of Military Medical Sciences, No. 8, Fengtaidongda Rd, Beijing I0007I, People's Republic of China Tel +86010 66947317 Fax +86010 66947317 Email 632763246@qq.com; Isb9126@126.com

\begin{abstract}
Percutaneous cementoplasty has been shown to immediately restore the mechanical stability of affected bones, prevent further risk of bone fractures, and allow immediate weight bearing. It is emerging as one of the most promising procedures for patients with painful bone metastasis who are unsuitable for surgery or who show resistance to radiotherapy and/or analgesic therapies. This study aimed at describing the procedure, indications, and benefits of percutaneous cementoplasty for painful osteolytic distal femur metastases. We report the case of a painful metastatic lesion in the left distal femur secondary to non-small-cell lung cancer in a 58-year-old woman. The patient underwent percutaneous cementoplasty and experienced effective pain relief and recovery of knee function postoperatively. In addition, no perioperative complication was observed. Percutaneous cementoplasty for osteolytic distal femur metastases offers effective pain relief and restores impaired knee function. Although this method may be a safe option, larger samples of retrospective or prospective confirmation are warranted.
\end{abstract}

Keywords: minimally invasive procedure, percutaneous cementoplasty, bone metastasis, distal femur

\section{Introduction}

Approximately $50 \%$ or more patients with cancer develop bone metastasis, and this is more common in patients with breast, prostate, and lung cancers. ${ }^{1,2}$ Bone metastasis often involves the vertebral column, pelvis, ribs, and proximal femur, whereas distal femur metastases are rare. The most frequent symptom is intense and drug-resistant pain that produces alterations in mobility and adversely and remarkably impacts the patient's quality of remaining life.

Currently, therapies for such patients include surgery, radiation therapy, chemotherapy, targeted therapy, immune therapy, and the use of bisphosphonates. ${ }^{3-7}$ However, pharmacological handling is insufficient to control pain, regain immediate bone integrity, and allow early weight bearing. Surgical intervention may be limited owing to high rates of complications and comorbidities. ${ }^{8}$ Therefore, a technique that can immediately restore the mechanical stability of the affected bones and allow immediate weight bearing is especially important. ${ }^{9,10}$

Fortunately, percutaneous cementoplasty, a new and minimally invasive technique, has been proven to be an effective therapy for bone metastatic lesions in the spine, pelvis, ilium, and proximal femur. ${ }^{9-15}$ It can alleviate pain ${ }^{9,10}$ and reduce metastatic activity ${ }^{16}$ while simultaneously offering stability to the affected bones. ${ }^{13-15}$ However, to the best of our knowledge, there has been no report thus far that addresses percutaneous 
cementoplasty for distal femur metastasis. Therefore, in this paper, we investigated the clinical benefits of this technique in distal femur metastasis with respect to pain relief, mobility improvement, and improved quality of life.

\section{Case report}

A 58-year-old woman who was diagnosed with non-small-cell lung cancer (stage IV) in March 2015 was referred in October 2015 to our department by her oncologist for severe knee pain and inability to ambulate. When the patient was initially diagnosed with lung cancer, opposite lung metastasis and rib metastasis had already occurred. The primary cancer was controlled with systematic chemotherapy. She described her knee pain as being 8 out of 10 on the visual analog scale (VAS), and her pain was drug resistant. Oxycodone hydrochloride prolonged-release tablets were administered at a dosage of $100 \mathrm{mg} / 12 \mathrm{~h}$, but there was no significant pain relief. Zoledronic acid was administrated at a dosage of $4 \mathrm{mg} / 4 \mathrm{wk}$, and her renal function was monitored. On physical examination, her left knee was grossly swollen, with generalized tenderness of the distal femur bone. Extension movements of her left knee would produce serious pain, and she was unable to stand or bear weight unaided. Radiography (Figure 1) confirmed the lytic destruction of the distal femur bone. Computed tomography showed the extent of the bone destruction, and magnetic resonance imaging showed the extent of the metastatic lesion (Figure 2). The patient did not have operative contraindications, such as local infection at the operation site, abnormal coagulation, and platelet dysfunction; hence, she was offered palliative radiotherapy, total knee arthroplasty, or an attempt at restoration by percutaneous cementoplasty as treatment

A

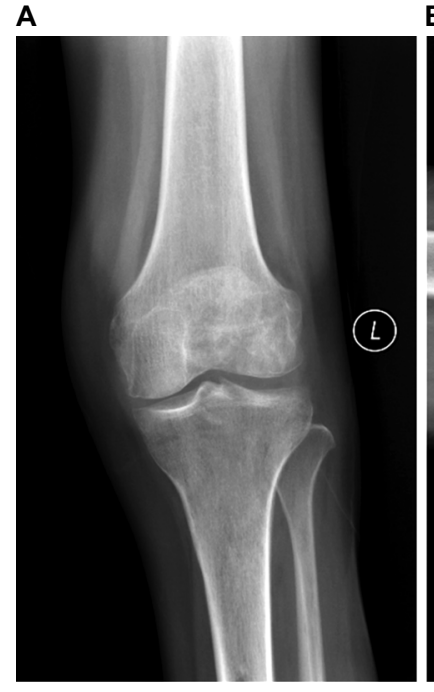

Figure I (A) Posteroanterior radiograph; (B) lateral radiograph confirming lytic destruction of the distal femur bone.

B

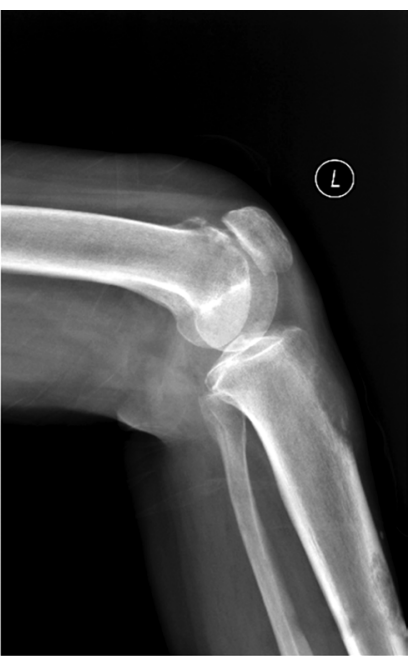

choices. She chose to undergo restoration incorporating polymethylmethacrylate cementoplasty.

This study was approved by the Medical Research Ethics Board of the Affiliated Hospital of Academy of Military Medical Sciences, and informed consent was obtained prior to performing all procedures. Written informed consent was obtained for publication of this paper and the accompanying images.

The patient was placed in the supine position. The patient's blood pressure, pulse, $\mathrm{PaO}_{2}$, and electrocardiogram were continuously monitored during the surgery. The operation site was infiltrated with $2 \%$ lidocaine + ropivacaine + normal saline (1.5:1:2). Under fluoroscopic guidance, a single 13-gauge needle was advanced into the metastatic lesion. Subsequently, we obtained metastatic tissues using a bone biopsy needle, and then injected the cement (approximately $6 \mathrm{~mL}$ ) through the 13-gauge needle under real-time fluoroscopic guidance. The filling was stopped when the distribution of cement in the metastatic lesion had been achieved. No complications occurred during the procedure.

We used the VAS, with scores ranging from 0 (indicating no pain) to 10 (indicating the greatest imaginable pain), to assess pain outcome. When the VAS score improved by three points or more, the procedure was considered effective. ${ }^{11}$ Mobility was assessed with the mobility scale, wherein a score of 1 represented no limitation, 2 represented limitation without the need for orthopedic aids, 3 represented limitation necessitating orthopedic aids, and 4 represented bedridden operative technique. ${ }^{17}$ Both the VAS and mobility scales were applied 24 hours before and after the procedure and at 1, 2, and 4 weeks and at 3 months after the procedure. The clinical complications that occurred during the procedure or within 24 hours after the procedure were documented in the report.

\section{Results}

Under local anesthetic, the patient was treated with percutaneous cementoplasty. No complications were observed during the operation and within 24 hours postoperatively. Her pain dropped from a preoperative VAS score of 8-6 within 24 hours postoperatively. Following surgery, analgesic drugs were administrated at a dosage of $10 \mathrm{mg} / 12 \mathrm{~h}$. Postoperative radiography showed cementation (Figure 3). The distal femur lesion was treated by regional radiotherapy 3 days after surgery. A bone biopsy 5 days after surgery confirmed metastasis resulting from lung adenocarcinoma. The patient was discharged from the hospital 1 week after the operation. At that time, she was non-weight-bearing and was able to move only with the use of a walker. One week after the operation, she described her pain as 3 out of 10 on the VAS. At the second week follow-up 

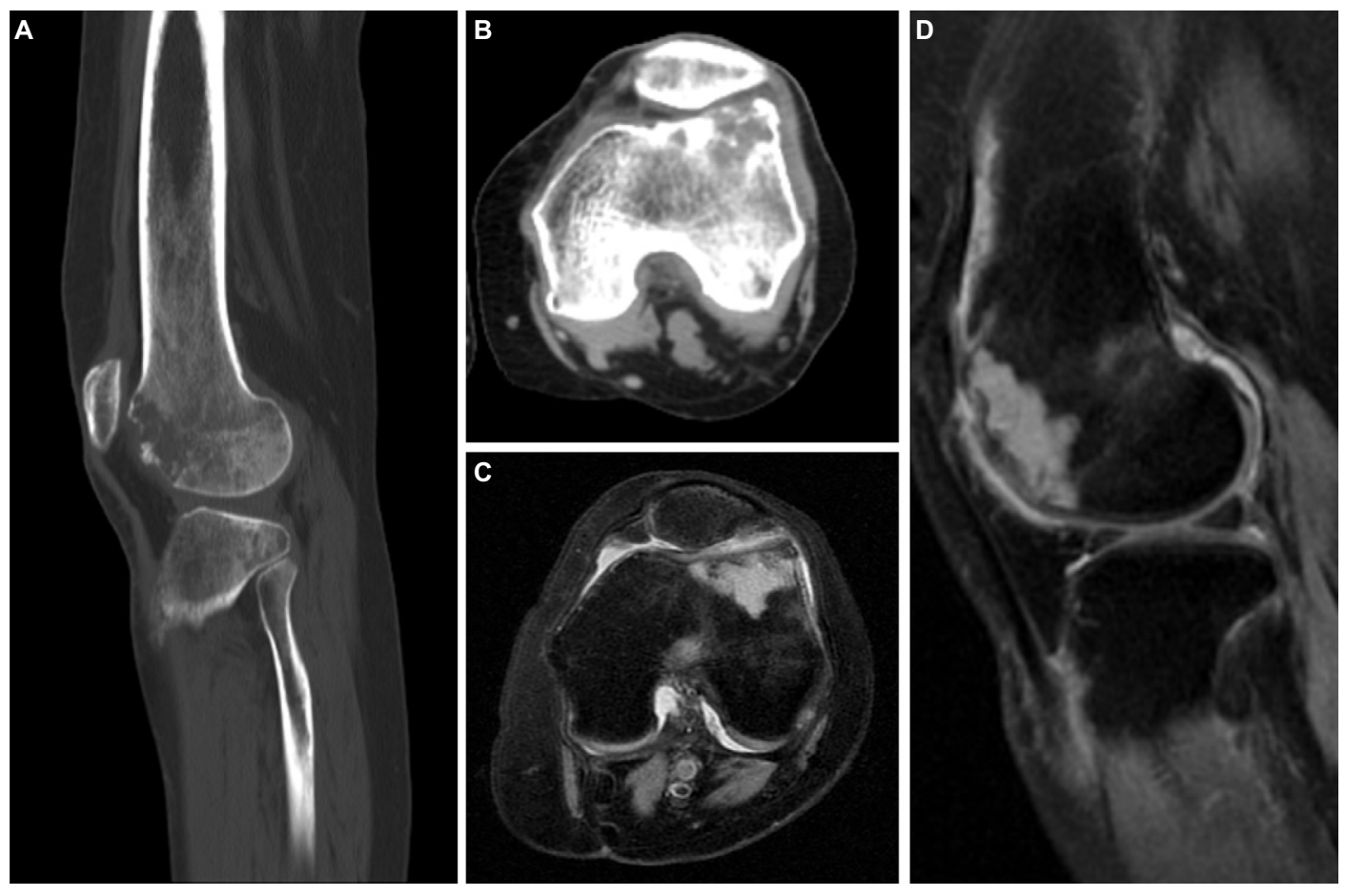

Figure 2 (A and B) CT shows the extent of bone destruction; (C and D) MRI shows the extent of the metastatic lesion. Abbreviations: $\mathrm{CT}$, computed tomography; MRI, magnetic resonance imaging.

A

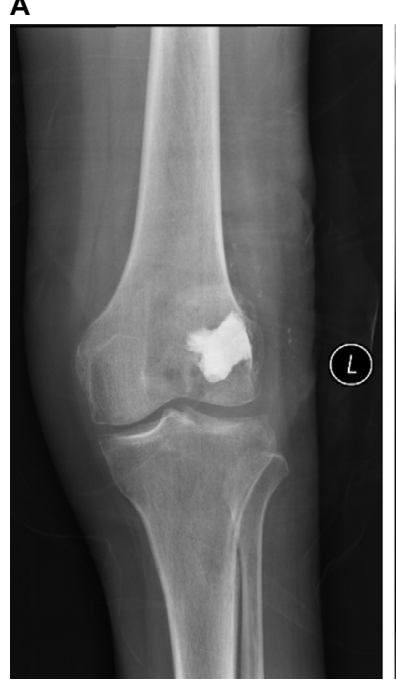

B

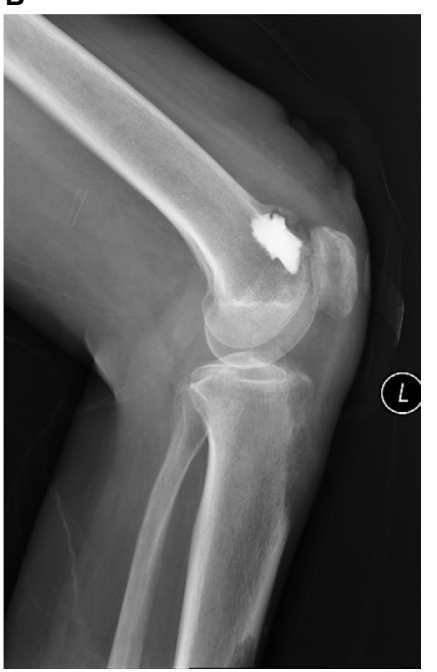

Figure 3 (A) Posteroanterior radiograph; (B) lateral radiograph showing the distribution of cement after percutaneous cementoplasty.

visit, the sutures were removed and full weight bearing was possible. Her VAS-based pain score remained steady at 3 out of 10 . At 4 weeks, her mobility had increased and she was able to walk across a corridor and go up and down the stairs without a walker. Meanwhile, the pain score further dropped from 3 to 2. Details of VAS and mobility scales are shown in Figure 4.

\section{Discussion}

The aim of treatment in patients with bone metastasis is to reduce pain, to maintain mobility, and to positively improve the patient's quality of remaining life. Currently, the therapies for these patients include surgery, radiation therapy, chemotherapy, targeted therapy, immune therapy, and the use of bisphosphonates. ${ }^{3,4}$ However, pharmacological handling is insufficient to control pain, immediately regain bone integrity, and allow early weight bearing. Surgical intervention may be limited on account of high rates of complication and comorbidities. ${ }^{8}$ Therefore, a treatment method that can provide pain control and improve bone stability at the same time is necessary.

Percutaneous cementoplasty can immediately restore the mechanical stability of the affected bone, prevent further risk of bone fractures, and allow immediate weight bearing. Therefore, it is emerging as one of the most promising procedures for patients with painful bone metastasis who are unsuitable for surgery or who show resistance to radiotherapy and/or analgesic treatment. ${ }^{9}$ Previous studies reported that $60 \%-84 \%$ of patients with metastatic compression fracture experienced marked or complete pain relief after vertebroplasty. ${ }^{10,11}$ In a multicenter randomized controlled trial, as compared with the nonsurgical group, the kyphoplasty group showed improved functional outcomes for patients with painful metastatic compression fracture, based on the Roland Morris disability questionnaire scores. ${ }^{12}$

Regarding femoral metastasis, Plancarte et $\mathrm{al}^{13}$ reported that all patients achieved pain reduction on the treated pelvic member, along with improved mobility, after femoroplasty 


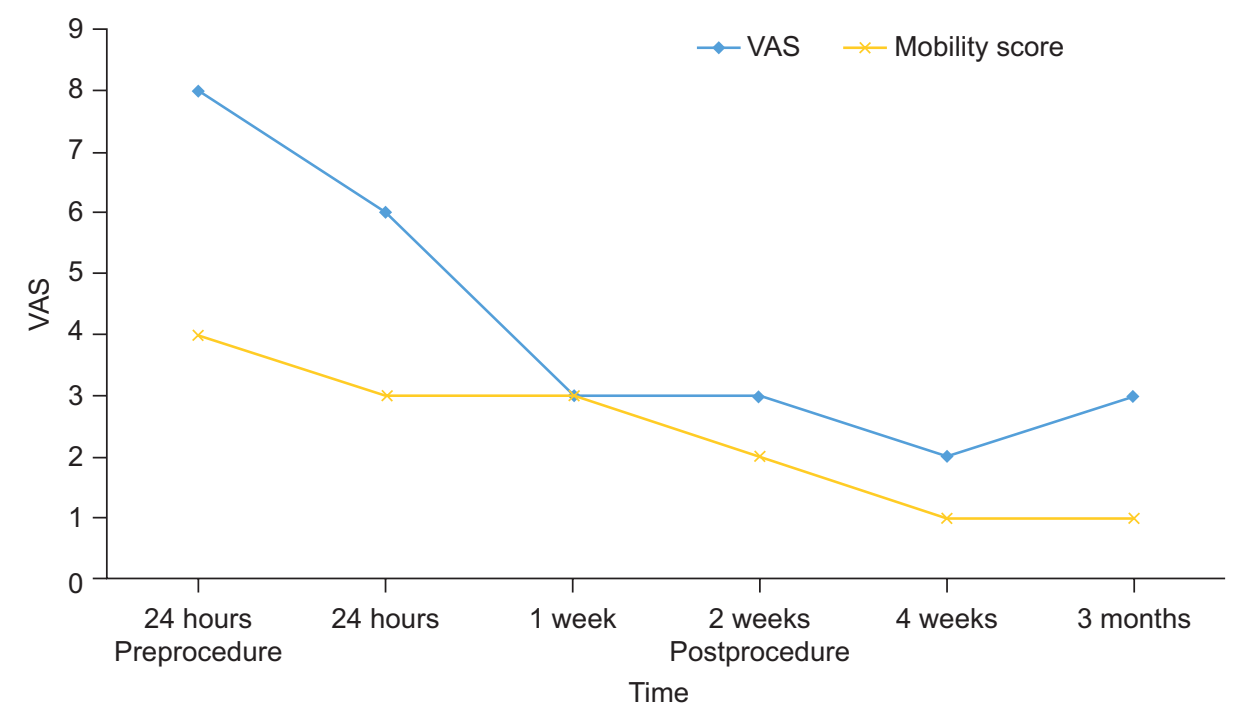

Figure 4 VAS and mobility scores before and after the procedure. Abbreviation: VAS, visual analog scale.

in a series of 15 patients with bone metastases located in the head, neck, and proximal femur. In a larger population, Plancarte et $\mathrm{al}^{14}$ also showed that all 80 patients had a decrease in the intensity of pain, reduced analgesic consumption, and improved quality of life at 7 and 30 days after the intervention, and there were no serious complications. In a systematic review of long bone tumors treated with percutaneous cementoplasty compiled by screening 1,598 articles and covering 196 patients, Cazzato et $\mathrm{al}^{15}$ found that $27.4 \%-68.2 \%$ patients showed pain improvement and 6\%-71.9\% patients obtained functional improvement. Cazzato et $\mathrm{al}^{18}$ also demonstrated in a series of 51 patients that percutaneous long bone cementoplasty was a safe and effective option. Local pain relief was observed in $89.4 \%$ lesions, and limb functionality was improved in $71.8 \%$ lesions. However, percutaneous cementoplasty in long bone is controversial. The primary argument has been that injecting cement directly into osteolytic lesions cannot provide adequate mechanical stability for long bones, which are considered to be very resistant to compressive forces but less resistant to torsional forces. ${ }^{19-21}$ Liu et $\mathrm{al}^{22}$ concluded that the efficacy of percutaneous long bone cementoplasty through embedding a cement-filled catheter in the medullary canal for painful long bone metastases with impending fracture was superior to percutaneous long bone cementoplasty alone in terms of pain relief, functional improvement, and the rate of fractures of the treated long bone. Deschamps et $\mathrm{al}^{23}$ also showed that percutaneous osteosynthesis plus cementoplasty was a promising method in the treatment of impending pathological fracture of the proximal femur. Overall, the combination of cementoplasty with other techniques produced encouraging results, but further investigations are still required for confirmation.
It has been suggested that when the polymethylmethacrylate bone cement is injected, it reconstructs stabilization of the destroyed bone and the microfracture, and the thermal action produced by the cement probably reduces the metastatic activity and inhibits the regional nociceptors, thus alleviating pain. ${ }^{16}$ In the present study, our patient was treated with percutaneous cementoplasty. No complications were observed during the operation and 24 hours postsurgery. Her pain decreased from a preoperative VAS score of 8 to 4 within 24 hours of the procedure. She was discharged from hospital 1 week after the operation. At that time, she was non-weight-bearing and used a walker for mobility; she described her postoperative pain score as 2 out of 10. At the 2 -week follow-up visit, full weight bearing was possible. At 4 weeks, her mobility had increased and she was able to go up and down stairs without using the walker. It is important to note that the patient's vital signs were continuously monitored during the procedure when we injected the cement so as to prevent related complications of cement leaking into the vessel.

\section{Conclusion}

In conclusion, percutaneous cementoplasty for osteolytic distal femur metastases is a relatively safe option, offering acceptable pain relief and restoring impaired knee function. We believe that this minimally invasive procedure is most suitable for patients having pain due to distal femur metastasis, thereby presenting a high risk of fractures of the involved femoral bone, or those with rapidly deteriorating quality of life. Nevertheless, for further confirmation, larger samples and retrospective or prospective studies are warranted. 


\section{Acknowledgment}

The work was supported by the Beijing Municipal Science and Technology Commission (No Z131107002213052 and No Z161100000516101), and Capital Health Research and Development Special Fund (No 2011-5006-03).

\section{Disclosure}

The authors report no conflicts of interest in this work.

\section{References}

1. Kuchuk I, Hutton B, Moretto P, Ng T, Addison CL, Clemons M. Incidence, consequences and treatment of bone metastases in breast cancer patients experience from a single cancer centre. J Bone Oncol. 2013;2(4):137-144.

2. Cetin K, Christiansen C F, Jacobsen J B, Nørgaard M, Sørensen HT. Bone metastasis, skeletal-related events, and mortality in lung cancer patients: a Danish population-based cohort study. Lung Cancer. 2014;86(2):247-254.

3. Ashford RU, Benjamin L, Pendlebury S, Stalley PD. The modern surgical and non-surgical management of appendicular skeletal metastases. Orthop Trauma. 2012;26(3):184-199.

4. Holen I, E Coleman R. Bisphosphonates as treatment of bone metastases. Curr Pharm Des. 2010;16(11):1262-1271.

5. Lei M, Liu Y, Tang C, Yang S, Liu S, Zhou S. Prediction of survival prognosis after surgery in patients with symptomatic metastatic spinal cord compression from non-small cell lung cancer. BMC Cancer. 2015;15:853.

6. Lei M, Li J, Liu Y, Jiang W, Liu S, Zhou S. Who are the best candidates for decompressive surgery and spine stabilization in patients with metastatic spinal cord compression (MSCC)? A new scoring system. Spine. Epub March 2, 2016.

7. Lei M, Liu Y, Liu S, Wang L, Zhou S, Zhou J. Individual strategy for lung cancer patients with metastatic spinal cord compression. Eur $J$ Surg Oncol. 2016;42(5):728-734.

8. Zhang X, Wu X, Zheng K, et al. Efficacy of surgical treatment strategies for long bone metastases. Support Care Cancer. 2015;23(1 Suppl):S33.

9. Wang Z, Zhen Y, Wu C, et al. CT fluoroscopy-guided percutaneous osteoplasty for the treatment of osteolytic lung cancer bone metastases to the spine and pelvis. J Vasc Interv Radiol. 2012;23(9):1135-1142.

10. Fourney D, Schomer D, Nader R, et al. Percutaneous vertebroplasty and kypho-plasty for painful vertebral body fractures in cancer patients. J Neurosurg. 2003;98(1):21-30.
11. Bae J, Gwak H, Kim S, et al. Percutaneous vertebroplasty for patients with metastatic compression fractures of the thoracolumbar spine: clinical and radiological factors affecting functional outcomes. Spine J. 2016;16(3):355-364.

12. Berenson J, Pflugmacher R, Jarzem P, et al. Balloon kyphoplasty versus non-surgical fracture management for treatment of painful vertebral body compression fractures in patients with cancer: a multicentre, randomised controlled trial. Lancet Oncol. 2011;12(3): 225-235.

13. Plancarte R, Guajardo J, Cerezo O, et al. Femoroplasty: a new option for femur metastasis. Pain Pract. 2013;13(5):409-415.

14. Plancarte R, Guajardo J, Meneses A, et al. Clinical benefits of femoroplasty: a nonsurgical alternative for the management of femoral metastases. Pain Physician. 2014;17(3):227-234.

15. Cazzato R, Palussière J, Buy X, et al. Percutaneous long bone cementoplasty for palliation of malignant lesions of the limbs: a systematic review. Cardiovasc Intervent Radiol. 2015;38(6):1563-1572.

16. Deramond H, Wleft NT, Belkoff SM. Temperature elevation caused by bone cement polymerization during vertebroplasty. Bone. 1999;25: 17S-21S.

17. Mpotsaris A, Abdolvahabi R, Hoffleith B, et al. Percutaneous vertebroplasty in vertebral compression fractures of benign or malignant origin: a prospective study of 1188 patients with follow-up of 12 months. Dtsch Arztebl Int. 2011;108(19):331-338.

18. Cazzato RL, Buy X, Eker O, Fabre T, Palussiere J. Percutaneous long bone cementoplasty of the limbs: experience with fifty-one non-surgical patients. Eur Radiol. 2014;24(12):3059-3068.

19. Gangi A, Buy X. Percutaneous bone tumor management. Semin Intervent Radiol. 2010;27(2):124-136.

20. Heini PF, Franz T, Fankhauser C, Gasser B, Ganz R. Femoroplastyaugmentation of mechanical properties in the osteoporotic proximal femur: a biomechanical investigation of PMMA reinforcement in cadaver bones. Clin Biomech. 2004;19(5):506-512.

21. Sutter EG, Mears SC, Belkoff SM. A biomechanical evaluation of femoroplasty under simulated fall conditions. J Orthop Trauma. 2010;24(2):95-99.

22. Liu XW, Jin P, Liu K, et al. Comparison of percutaneous long bone cementoplasty with or without embedding a cement-filled catheter for painful long bone metastases with impending fracture. Eur Radiol. Epub April 21, 2016.

23. Deschamps F, Farouil G, Hakime A, Teriitehau C, Barah A, de Baere T. Percutaneous stabilization of impending pathological fracture of the proximal femur. Cardiovasc Inter Rad. 2012;35(6):1428-1432.
Journal of Pain Research

\section{Publish your work in this journal}

The Journal of Pain Research is an international, peer reviewed, open access, online journal that welcomes laboratory and clinical findings in the fields of pain research and the prevention and management of pain. Original research, reviews, symposium reports, hypothesis formation and commentaries are all considered for publication.

\section{Dovepress}

The manuscript management system is completely online and includes a very quick and fair peer-review system, which is all easy to use. Visit http://www.dovepress.com/testimonials.php to read real quotes from published authors. 